\title{
Influencia de las TIC en el rendimiento escolar de estudiantes vulnerables
}

\section{(Influence of ICT on the school performance of vulnerable students)}

\author{
Inés María González Vidal \\ Universidad Santiago de Compostela, USC (España)
}

DOI: http://dx.doi.org/10.5944/ried.24.1.27960

\section{Cómo referenciar este artículo:}

Vidal, I. M. G. (2021). Influencia de las TIC en el rendimiento escolar y su impacto en estudiantes vulnerables. RIED. Revista Iberoamericana de Educación a Distancia, 24(1), pp. 351-365. doi: http://dx.doi.org/10.5944/ried.24.1.27960

\section{Resumen}

El sistema educativo contemporáneo está influenciado por los movimientos migratorios y el desarrollo de las Tecnologías de la Información y la Comunicación (TIC). Este trabajo analiza la influencia de las TIC en el rendimiento escolar de estudiantes vulnerables. Para ello, se estudian las relaciones del rendimiento escolar con el país de origen del estudiante, la conexión a Internet en el hogar y el uso diario de dispositivos digitales. Apoyado en la metodología de investigación en educación comparada, se contrastan las muestras representativas de las poblaciones de España, los países miembros de la Unión Europea y la Organización para la Cooperación y el Desarrollo Económicos. Se utilizan fundamentalmente los datos publicados por el Ministerio de Educación en los informes PISA 2018 y OCDE 2019. La estadística descriptiva sustenta los resultados obtenidos. Se analizan las variables participación diaria en redes sociales, participación diaria en juegos online, lectura diaria de noticias online y la nota media de Ciencias y Matemática respectivamente. Los hallazgos muestran que en España existe un bajo rendimiento escolar relacionado con el origen del estudiante; la conexión a Internet en el hogar y que un uso diario de dispositivos digitales puede ser beneficioso, si las exposiciones son moderadas y controladas.

Palabras clave: internet; estudiantes; inmigración; redes sociales; juegos online; educación secundaria obligatoria. 


\begin{abstract}
The contemporary educational system is influenced by migratory movements, as well as the development of Information and Communication Technologies (ICT). The goal of the work is to analyze the influence of ICT on the school performance of vulnerable students. To do this, the relationships between school performance and the student's country of origin, the Internet connection at home and the daily use of digital devices are studied. Supported by the comparative education research methodology, representative samples of the populations of Spain, the member countries of the European Union and the Organisation for Economic Co-operation and Development are contrasted. Descriptive statistics support the results obtained. The variables daily participation in social networks, daily participation in online games, daily reading of online news, and the average grade for Science and Mathematics, respectively, are analyzed. The findings show that in Spain there is low school performance related to the origin of the student; Internet connection at home and that daily use of digital devices can be beneficial, if the exposures are moderate and controlled.
\end{abstract}

Keywords: internet; students; immigration; social networks; online games; secondary education.

El sistema educativo contemporáneo, está fuertemente influenciado por el desarrollo vertiginoso de las TIC (Tecnologías de la Información y las Comunicaciones), así como por el proceso de globalización y movimientos migratorios (Dryden-Peterson, 2016; Caamaño, 2009). Vivimos en una sociedad del conocimiento y los entornos virtuales generan gran cantidad de información, todo esto es aprovechado para perfeccionar la educación (Leicht, Heiss y Byun, 2018).

En este sentido, el desarrollo de las TIC va unido a las nuevas tendencias innovadoras en la educación y causa avances significativos en el proceso de enseñanza y aprendizaje (Martín-Gutiérrez, Mora, Añorbe-Díaz y González-Marrero, 2017). Por ejemplo, los estudiantes tienen acceso a una variedad de recursos tecnológicos que los mantiene conectados a Internet todo el tiempo (Bulman y Fairlie, 2016). Esta peculiaridad es aprovechada para permitirles organizar el proceso de aprendizaje acorde a sus necesidades individuales e intereses profesionales además de tener gran influencia en los resultados docentes (Hurwitz y Schmitt, 2020). Del mismo modo, el uso creciente de técnicas propias de los juegos en entornos virtuales como pre-concepción del aprendizaje, potencia la motivación del estudiante hacia nuevos contenidos educativos y sirve como predictor del éxito académico y diversas habilidades (Werbach y Hunter, 2015).

Dentro de este orden de ideas, el uso de las TIC incrementa las experiencias de intercambio de actividades educativas, diversificando y personalizando la enseñanza (Echazarra, 2018); se rompen las barreras de espacio y tiempo, así como las distancias físicas y culturales (Castañeda Quintero y Adell, 2013; Terrén, 2007). En definitiva, los estudiantes se convierten en participantes activos en la construcción del 
conocimiento y pueden acceder, almacenar, reutilizar, crear y compartir contenidos y recursos virtuales (Torres Kompen y Costa, 2013).

Por otro lado, el sistema educativo contemporáneo está caracterizado por entornos con gran pluralismo étnico o cultural (Schachner, 2019). Ante esta situación, los gobiernos implementan nuevas medidas relacionadas con las políticas de integración de estudiantes inmigrantes en las sociedades de acogida (Marks, McKenna y Coll, 2018). Estas políticas propugnan la convivencia intercultural y multicultural basada en la empatía, la tolerancia y el respeto a la diversidad (Lieberman y Gamst, 2015).

Cabe destacar que la integración exitosa comienza por el sistema educativo y esta situación se enfrenta de manera diferente en cada país (Coin, 2017; Kogan, 2016), también guarda relación con el nivel de desarrollo económico del país de origen (Huddleston, Niessen y Tjaden, 2013). En otras palabras, la integración educacional es una referencia importante en la integración de estudiantes inmigrantes en las sociedades de acogida (Zubíková, 2019). No obstante, existen barreras relacionadas con la adquisición de la lengua del país de acogida, la cultura y la falta de formación del profesorado para enfrentar estos nuevos retos (Esteban, 2020; Manning y Roy, 2010).

Contextualizando, en España algunos programas caracterizan al estudiantado inmigrante como un colectivo homogéneo, aplicando soluciones iguales a todos, sin conseguir promover su inclusión socioeducativa e intercultural (Valero y Viñas, 2019). Estos programas no consideran la participación e implicación familiar (Moledo, Rego y Otero, 2012), el rendimiento escolar y la etapa evolutiva del menor (Caamaño, 2013). Cabe resaltar que otros estudios consideran que la ayuda al alumnado inmigrante se debe proporcionar desde dentro del aula ordinaria, en el marco de una escuela inclusiva (Balerdi, Larrañaga, Cartón y Aranguren, 2018).

En relación a la problemática anteriormente expuesta, se visualiza la necesidad de implementar estrategias efectivas de intervención para mejorar los servicios de apoyo a estudiantes vulnerables y visualizar factores que puedan incidir en el éxito y fracaso académico (Spörlein y Schlueter, 2018), analizar la preparación previa, así como otros factores que puedan influir en el rendimiento académico (Belvis, Moreno y Ferrer, 2009).

En este panorama, se vislumbra la ausencia de consenso respecto del significado de la integración en la educación y de las formas para alcanzarla con respeto a la diversidad cultural del estudiantado (Goicochea, 2008). En este sentido, la literatura científica reconoce la necesidad impostergable de incrementar las investigaciones sobre la influencia de las TIC en el rendimiento escolar para minimizar su impacto en estudiantes vulnerables y facilitar el proceso de integración de estudiantes inmigrantes en el sistema educativo (Caamaño, 2009).

El objetivo de este trabajo es analizar la influencia de las TIC en el rendimiento escolar de estudiantes vulnerables. La metodología de investigación utilizada es en educación comparada. La principal contribución de este trabajo en el contexto educativo español, es referido al rendimiento escolar relacionado con el país de 
origen del estudiante y de la importancia de la implementación de mejores políticas de integración educacional. La segunda contribución está relacionada con la conexión a Internet en el hogar y que un uso diario de dispositivos digitales puede ser beneficioso para el estudiante si las exposiciones son moderadas y controladas.

\section{MATERIALES Y MÉTODOS}

La metodología en educación comparada, es el campo disciplinar que emplea como procedimiento de investigación el método comparatista, es decir, engloba la descripción - interpretación - yuxtaposición - comparación como niveles de análisis (Adick, 2018). El comparatismo se utiliza en el área educativa como una operación metodológica, relacional y crítica con implementación tecnológica (Martí Marco, 2019). La primera operación comparatista es la delimitación de los objetos a comparar (Mombelli, 2019).

Este estudio pretende analizar la influencia de las TIC en el rendimiento escolar de estudiantes vulnerables. Se realiza un análisis comparativo del rendimiento escolar con el país de origen del estudiante, la conexión a Internet en el hogar y el uso diario de dispositivos digitales. Se trabaja con una muestra representativa recogida de las poblaciones de España, los países miembros de la UE (Unión Europea) y la OCDE (Organización para la Cooperación y el Desarrollo Económicos). Para ello, se utilizan fundamentalmente los datos publicados por el Ministerio de Educación en los informes respectivos PISA 2018 (Cebrián et al., 2019) y OCDE 2019 (González Merino, 2020) ${ }^{1}$, aunque también se referencian informes de años anteriores y otras fuentes de información. La muestra de estudiantes objeto de estudio pertenece a una franja etaria de entre 15 y 16 años, que estudian el décimo grado o cuarto curso de Educación Secundaria Obligatoria en España (Cebrián, Trillo y González, 2019).

A partir del análisis documental se identifican relaciones entre las variables rendimiento escolar y el país de origen del estudiante. Se define la variable cualitativa ordinal conexión a Internet en el hogar que toma distintos valores ordenados siguiendo una escala establecida, a saber, acceso excesivo, sin acceso y acceso moderado. El uso diario de dispositivos digitales es una variable compleja compuesta por las siguientes dimensiones: la participación diaria en redes sociales, la lectura diaria de noticias online, y la participación diaria en juegos online, así como las notas medias de Ciencias y Matemáticas respectivamente.

La comprensión lectora en el estudio PISA 2018 (Cebrián et al., 2019) fue una competencia principal de evaluación. No obstante, en este trabajo no se consideran los resultados directamente, debido a la propia naturaleza compleja de la lectura, y la creciente influencia de las tecnologías de la información y de la comunicación en esta variable.

Para el análisis del uso diario de dispositivos digitales en su dimensión participación diaria en redes sociales se aplican estadísticos descriptivos. El primer estadístico es un estudio de inferencia a través de una prueba de hipótesis 
Chi cuadrado, en general esta prueba contrasta frecuencias observadas con las frecuencias esperadas de acuerdo con la hipótesis nula. El segundo estadístico es un modelo de regresión lineal o ajuste usado para aproximar la relación de dependencia entre una la variable independiente $\mathrm{X}$ y la variable dependiente $\mathrm{Y}$, así como un término aleatorio $\varepsilon$. Si bien cabe destacar el reducido número de datos utilizados, los resultados obtenidos son preliminares y constituyen el punto de partida para trabajos futuros.

\section{DISCUSIÓN Y RESULTADOS}

\section{Análisis del Rendimiento y origen del estudiante}

Estudios anteriores, relacionan el bajo rendimiento escolar de los estudiantes inmigrantes frente a los nativos, indicando una relación entre el rendimiento escolar y las circunstancias sociodemográficas del estudiante (OCDE, 2016). Otros estudios señalan que en España, los estudiantes inmigrantes que no proceden de los países de la UE obtienen los peores valores de rendimiento escolar, el factor individual juega un papel importante en este sentido (Contucci y Sandell, 2015).

En España la tasa de abandono escolar temprano es de un 35,7\% en estudiantes inmigrantes, frente al $14,7 \%$ en sus colegas nativos y estos resultados fueron relacionados con el nivel educativo de los padres (Cebrián et al., 2019). Los estudiantes inmigrantes en España obtienen 34 puntos menos en la nota media de Ciencias y 40 puntos menos en la nota media de Matemática, comparado con la media OCDE frente a sus colegas nativos en el nivel educativo de cuarto curso de Educación Secundaria Obligatoria (Cebrián et al., 2019).

La proporción de estudiantes inmigrantes varía por regiones en España. Los valores más bajos de proporción de estudiante inmigrantes frente nativos, con una proporción que no llega a 1 de cada 10 estudiantes, lo encontramos en las Comunidades Autónomas de Castilla y León (9\%), Asturias (8,3\%), Cantabria (8,3\%), Andalucía (6,6\%), Galicia (6,4\%), entre los ciclos de PISA 2015 (Gurría, 2016) y PISA 2018 (Cebrián et al., 2019).

Las diferencias de rendimiento medio en Ciencias entre estudiantes inmigrantes y nativos se hicieron más evidentes superando los 45 puntos en Castilla y León (49 puntos), Región de Murcia (52 puntos), País Vasco (55 puntos) y Galicia (61 puntos). Sin embargo, en Canarias (20 puntos) y en Andalucía (20 puntos), estas diferencias no son relevantes. No se apreciaron diferencias significativas en el rendimiento en Ciencias entre estudiantes nativos e inmigrantes en Ceuta y Melilla según informe PISA 2018 (Cebrián et al., 2019).

Resulta necesario señalar, que en países como Austria, Nueva Zelandia y Canadá, con alta proporción de estudiantes inmigrantes, el rendimiento de estudiantes inmigrantes es igual o superior al de sus compañeros nativos, en definitiva "a pesar de las diferencias observadas, en muchos países con una proporción considerable 
de estudiantes inmigrantes son capaces de alcanzar al menos unos niveles mínimos de rendimiento, superando sus numerosas condiciones desfavorables de partida" (Cebrián et al., 2019, p.99).

\section{Disponibilidad de conexión de Internet en el hogar}

Algunos autores relacionan la conexión a Internet en el hogar con el rendimiento escolar de los estudiantes (Cebrián et al., 2019). La figura 1 representa un resumen del análisis documental realizado referido a la influencia de la disponibilidad y/o conexión a Internet en el hogar y los resultados docentes del estudiante.

En tal caso, el uso responsable y seguro de dispositivos digitales con conexión a Internet en el hogar permite acceder a mayor cantidad de información digital (Hurwitz y Schmitt, 2020). No obstante, el acceso excesivo a Internet provoca una disminución del tiempo dedicado al estudio, y repercute en el rendimiento escolar con peores resultados docentes (Díaz-Vicario, Mercader Juan y Gairín Sallán, 2019).

Figura 1. Análisis de la disponibilidad y/o conexión a Internet en el hogar.

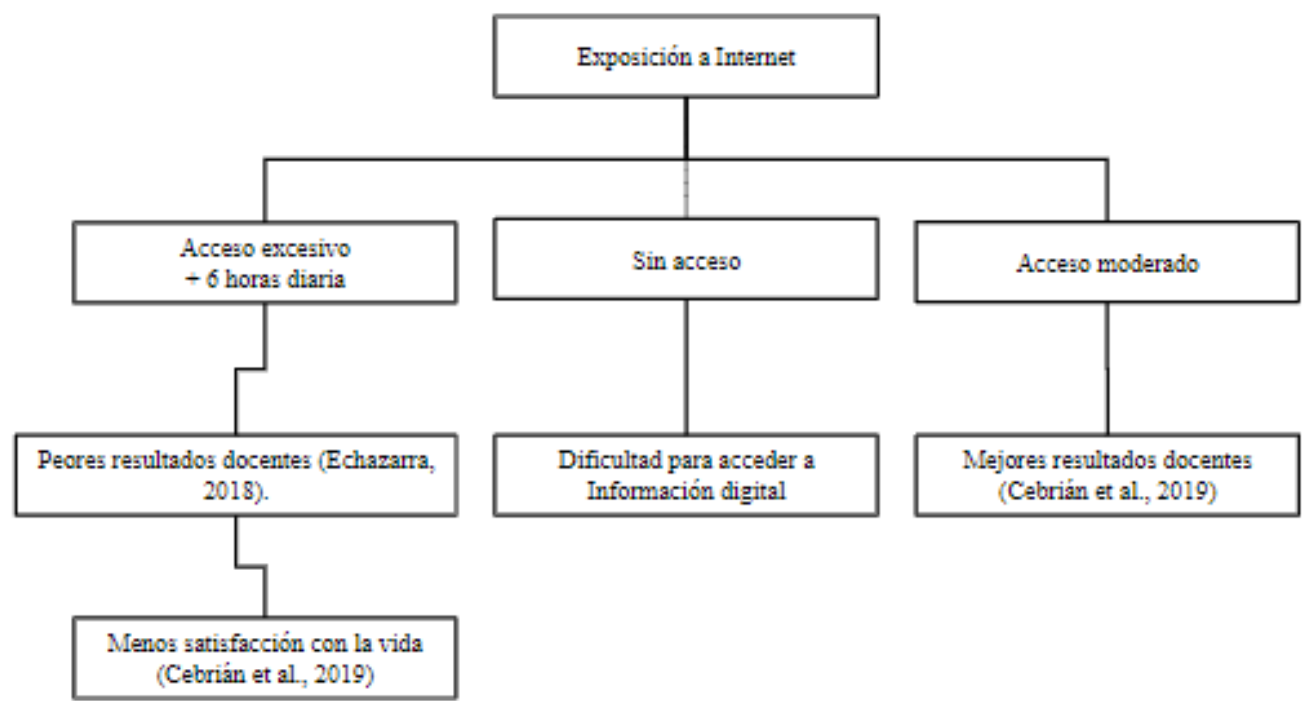

Fuente: Elaboración propia a partir de PISA 2018 (Cebrián et al., 2019).

En otro sentido, los estudiantes que no tienen acceso a Internet en el hogar, van a presentar dificultad para obtener la información digital, no podrán realizar actividades online desde el hogar, por consiguiente, su rendimiento escolar se verá afectado (Rodicio-García, Ríos-de-Deus, Mosquera-González y Abilleira, 2020). En 
consecuencia, estos alumnos no consiguen avanzar al mismo ritmo que los que sí están conectados desde sus hogares.

Cabe adicionar, que en España 11,9 \% de niños entre 10 y 15 años no utilizan Internet en sus hogares, según datos del informe Datos y cifras curso escolar 2019/2020 (Secretaría General Técnica, 2020, p.33). En relación con las implicaciones, se visualiza la vulnerabilidad de un grupo de estudiantes frente a los nuevos escenarios y retos educativos provocados por la crisis sanitaria COVID-19 en el curso 2019-2020.

\section{Uso diario de dispositivos digitales}

En este análisis, se realiza un estudio comparativo con datos referidos al uso diario de dispositivos digitales, se trabaja con una muestra representativa recogida de las poblaciones de España, los países miembros de la UE y la OCDE. (Cebrián et al., 2019, p. 128). Como el uso diario de dispositivos digitales es una variable compleja, se definen varias dimensiones en este análisis: la participación diaria en redes sociales (PDRS), la lectura diaria de noticias online (LDNO), y la participación diaria en juegos online (PDJO), así como las notas medias de Ciencias (NMC) y Matemáticas (NMM) respectivamente.

En la tabla 1 (a) se muestra la tabla de frecuencias que representa a las variables participación diaria en redes sociales (PDRS), nota media de Ciencias (NMC) y nota media en Matemáticas (NMM). Los datos mostrados evidencian, que los estudiantes españoles tienen el mayor valor de PDRS en relación a los estudiantes de los países miembros de la UE y la OCDE. Además, poseen los peores valores de NMC y NMM respectivamente. A primera vista se puede pensar que cuando disminuye la PDRS aumentan las NMC y NMM respectivamente.

El primer estadístico que se utiliza para demostrar el planteamiento anterior, es un estudio de inferencia a través de una prueba de hipótesis Chi-cuadrado, ver tabla 1 (b), siendo la variable independiente PDRS y la variable dependiente NMC (NMM respectivamente). La hipótesis nula Ho que se plantea es que no existe dependencia entre las variables PDRS y NMC (NMM respectivamente) la hipótesis alternativa H1 será que existe dependencia entre las variables.

El contraste Chi-cuadrado para un $\alpha$ (alfa) $=0,05$ proporciona un p-valor de 0,2028 (o,173 respectivamente), como el valor $\mathrm{P}$ no es inferior a $\alpha$, entonces la hipótesis nula no se rechaza. Como este resultado es muy sorprendente, no se sacan conclusiones apoyándonos exclusivamente en el p-valor, debemos tener en cuenta también estudios anteriores y quizás no emitir un juicio definitivo siendo tal resultado denominado estadísticamente significativo. 
Tabla 1. Resumen estadístico para PDRS en su relación con NMC (NMM respectivamente).

\begin{tabular}{|l|c|c|c|}
\hline \multicolumn{4}{|c|}{ Tabla de frecuencias } \\
\hline & PDRS & NMC & NMM \\
\hline OCDE & 107 & 490 & 494 \\
\hline UE & 127 & 489 & 489 \\
\hline ESPAÑA & 136 & 483 & 481 \\
\hline
\end{tabular}

a)

\begin{tabular}{|l|c|c|}
\hline \multicolumn{3}{|c|}{ Prueba de hipótesis (Chi cuadrada) } \\
\hline & PDRS/NMC & PDRS/NMM \\
\hline Alfa & 0,05 & 0,05 \\
\hline df & 2 & 2 \\
\hline Valor P & 0,2027 & 0,1730 \\
\hline Estadística de prueba & 3,1913 & 3,5088 \\
\hline Valor crítico & 5,9914 & 5,9914 \\
\hline
\end{tabular}

b)

\begin{tabular}{|c|c|c|c|}
\hline \multicolumn{3}{|c|}{ Modelo de regresiónLineal } \\
\hline \multicolumn{2}{|c|}{ PDRS/NMC } & \multicolumn{2}{c|}{ PDRS/NMM } \\
\hline $\begin{array}{c}\text { Salida bruta de } \\
\text { estimación lineal }\end{array}$ & $\begin{array}{c}\text { Salida bruta de } \\
\text { estimación lineal }\end{array}$ \\
\hline$-0,2095$ & 513,1754 & $-0,4152$ & 539,2178 \\
\hline 0,1454 & 18,0226 & 0,1506 & 18,6721 \\
\hline 0,6748 & 3,0528 & 0,8836 & 3,1628 \\
\hline 2,0758 & 1 & 7,5967 & 1 \\
\hline 19,3466 & 9,319 & 75,9962 & 10,0037 \\
\hline
\end{tabular}

c)

\begin{tabular}{|l|c|c|}
\hline \multicolumn{3}{|c|}{ Estadísticas de regresión lineal } \\
\hline $\mathrm{R}^{2}$ & PDRS/NMC & PDRS/NMM \\
\hline Error estándar & 0,6748 & 0,8836 \\
\hline Recuento de variables X & 3,05286 & 3,1628 \\
\hline Observaciones & 1 & 1 \\
\hline $\mathrm{R}^{2}$ ajustado & 3 & 3 \\
\hline Nivel de confianza & 0,3497 & 0,7673 \\
\hline
\end{tabular}

d)

Fuente: Elaboración propia con estadística LibreOffice Cal. 
Un segundo estadístico que se aplica, es un modelo de regresión lineal o ajuste usado para aproximar la relación de dependencia entre una variable dependiente $\mathrm{Y}$, la variable independientes $\mathrm{X}$ y un término aleatorio $\varepsilon$.

La tabla 1 (c) representan la generación de modelos de regresión lineal que precede al análisis de la estadística de regresión, ver la tabla 1 (d). A la luz de los resultados, se observa que la PDRS guarda una relación inversa con la NMC (NMM respectivamente), el valor de declive es una pendiente negativa -0, $2095(-0,4153)$. La respuesta en este caso, pudiera explicar la relación entre las variables PDRS y NMC (NMM respectivamente) a través de coeficientes determinación, en este caso $\mathrm{R}^{\wedge} 2=67,49 \%(88,37 \%$ respectivamente). El R2 es el porcentaje de variación de la variable de respuesta que explica su relación con la variable predictora. Por lo general, mientras mayor sea el R2, mejor será el ajuste del modelo a sus datos.

Las figuras 2 (a) y (b) por su lado, muestran las líneas de regresión negativa, y esta tendencia es confirmada por el signo negativo de las pendientes de las ecuaciones de la línea de tendencia en ambos casos.

Figura 2. Gráficos de dispersión con líneas y ecuaciones de tendencia

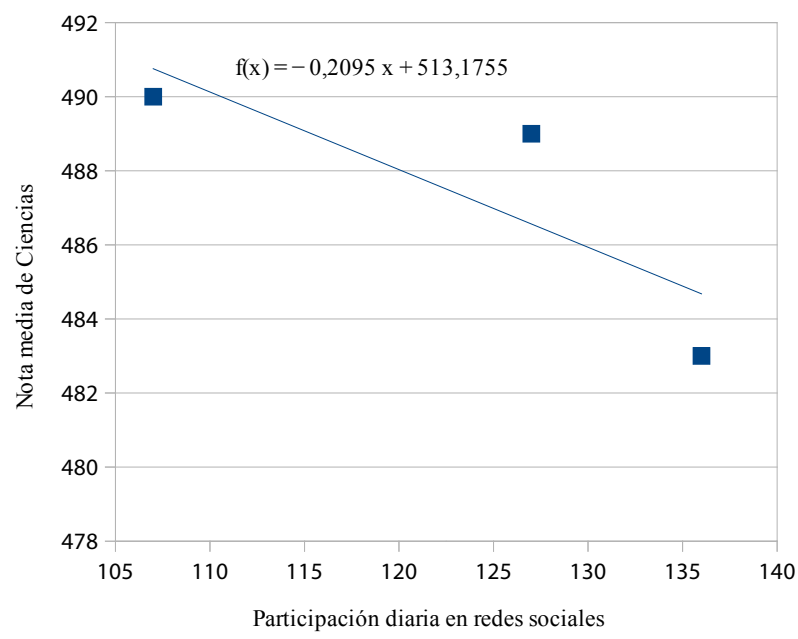

a) Caso PDRS y NMC 


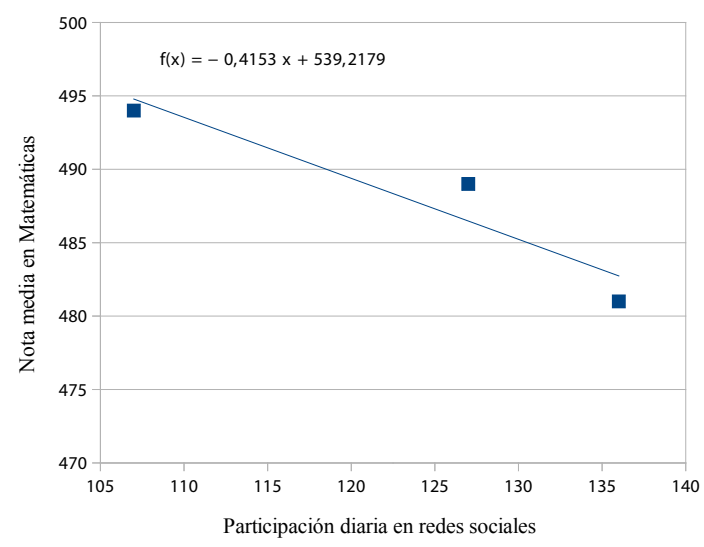

b) Caso PDRS y NMM.

Fuente: Elaboración propia con estadística LibreOffice Cal.

La lectura diaria de noticias online en los estudiantes está motivada por tres factores fundamentales: el monitoreo de la actualidad, el entretenimiento y la utilidad social (Condeza, Bachmann y Mujica, 2014). La tabla 2 muestra las frecuencias correspondientes a las variables lectura diaria de noticias online (LDNO), la nota media de Ciencia (NMC) y la nota media de Matemáticas NMM.

Tabla 2. Tabla de frecuencias para LDNO en su relación con NMC (NMM respectivamente).

\begin{tabular}{|l|c|c|c|}
\hline \multicolumn{4}{|c|}{ Tabla de frecuencias } \\
\hline OCDE & LDNO & NMC & NMM \\
\hline UE & 31 & 490 & 494 \\
\hline España & 46 & 489 & 489 \\
\hline
\end{tabular}

Fuente: Elaboración propia con estadística LibreOffice Cal.

Los datos indican que los estudiantes españoles presentan los valores más bajos de LDNO frente a sus colegas de países miembros de la UE y la OCDE. Si bien cabe destacar el reducido número de datos utilizados, estos no describen a simple vista una relación entre la LDNO y la NMC (NMM respectivamente).

No obstante, trabajos similares manifiestan que la lectura por placer y la situación socioeconómica y cultural de la familia son las variables que tienen mayor efecto positivo sobre las notas de los alumnos, todo esto está relacionado con el adecuado uso de las TIC y la disponibilidad de dispositivos digitales en el hogar (Fuentes y Gutiérrez, 2013). 
Tabla 3. Tabla de frecuencia para PDJO en su relación con NMC (NMM respectivamente)

\begin{tabular}{|l|c|c|c|}
\hline \multicolumn{4}{|c|}{ Tabla de frecuencias } \\
\hline & PDJO & NMC & NMM \\
\hline OCDE & 32 & 490 & 494 \\
\hline UE & 36 & 489 & 489 \\
\hline ESPAÑA & 24 & 483 & 481 \\
\hline
\end{tabular}

Fuente: Elaboración propia con estadística LibreOffice Cal.

La tabla 3 muestra las frecuencias de las variables participación diaria en juegos online (PDJO), la nota media en Ciencias (NMC) y nota media de Matemática (NMM). Se observa que la cantidad de estudiantes españoles que declaran participar diariamente en juegos online es menor que en los países miembros de la UE y la OCDE. Estos datos no describen a simple vista una relación entre la PDJO y la NMC (NMM respectivamente).

No obstante, otros estudios similares analizan los riesgos y los patrones de juego e identifican relaciones significativas entre el control parental y el rendimiento académico, en este sentido un mayor número de horas de juego se relaciona con un bajo rendimiento escolar (Irles, Perona y Baños, 2015).

\section{CONCLUSIONES}

El primer aspecto analizado en este trabajo fue la relación entre rendimiento escolar y el país de origen del estudiante. Al respecto se visualiza que en España existe un bajo rendimiento escolar relacionado con el país de origen del estudiante, es decir los estudiantes inmigrantes mostraron un rendimiento inferior frente a sus colegas nativos. Cabe destacar que el abandono escolar temprano en España se relaciona con el nivel educativo de sus padres, por tanto, la influencia de la situación socioeconómica y cultural de la familia tiene efecto en el rendimiento escolar del estudiante. En definitiva, se evidencian la existencia de una brecha social en la Educación, y la necesidad de la implementación de mejores políticas de integración educacional que podrían ayudar a disminuir estas diferencias aún presentes en algunas comunidades autónomas españolas.

Otro punto analizado fue la relación del rendimiento escolar y la conexión a Internet. En este sentido, la disponibilidad de conexión a Internet en el hogar permite el acceso a contenidos digitales que pueden ser beneficiosos para que los estudiantes obtengan buenos resultados docentes, siempre que las exposiciones sean moderadas y controladas. No obstante, las exposiciones excesivas a Internet de manera improductiva, no conducen al crecimiento de las habilidades digitales, de modo que se pierden oportunidades educativas potencialmente beneficiosas. Por otro lado, los estudiantes que no tienen acceso a Internet en el hogar no podrán acceder a una 
educación virtual, van a presentar dificultad para obtener la información digital, no podrán realizar actividades online desde el hogar, y por consiguiente, su rendimiento escolar se verá afectado. En definitiva, este trabajo visualiza la presencia en España de una brecha digital en un grupo de estudiantes vulnerables frente a los nuevos escenarios y retos educativos provocados por la crisis sanitaria COVID-19 en el curso 2019-2020

Por último, se estudia la relación del rendimiento escolar y el uso diario de dispositivos digitales. En este sentido el uso diario de dispositivos digitales favorece el cambio de rutinas y hábitos en los estudiantes, constituye una nueva forma para acceder a las informaciones, y en consecuencia este aspecto puede guardar relación con el rendimiento escolar de los estudiantes. En este sentido se consideró el uso diario de dispositivos digitales una variable compleja, compuesta por tres dimensiones, los resultados fueron los siguientes: 1- el uso excesivo de las redes sociales puede generar impactos negativos en el ámbito académico y por ende, repercutir en el rendimiento escolar con peores resultados docentes, 2- la lectura diaria de noticias online tiene una influencia positiva en el rendimiento escolar del estudiante, 3- un mayor número de horas de juego se relaciona con un bajo rendimiento escolar, por esta razón, se debe promover un modelo de juego seguro y controlado.

A modo de cierre, nuevos escenarios influencian el sistema educativo, a saber, el desarrollo vertiginoso de las TIC aplicadas a la educación, los procesos de globalización y los movimientos migratorios. En consecuencia, un impulso a la educación online se abre con un lente crítico que permite la reflexión sobre los nuevos retos que se exigen en la sociedad contemporánea. Paralelamente, surge la necesidad de incrementar las investigaciones para allanar el camino y promover la adopción de nuevas soluciones que permitan el perfeccionamiento de la educación virtual. En este sentido, las instituciones y los gobiernos deben adaptarse rápidamente y adoptar nuevas políticas de integración que garanticen la equidad y la innovación en la educación. En este escenario, las TIC tienen un impacto decisivo en las transformaciones de la dinámica educativa, que rebasa la mera experiencia intercultural persona a persona y la reemplaza por una experiencia de conectividad intercultural.

\section{NOTAS}

1. La OCDE es una Organización para la Cooperación y el Desarrollo Económicos, es un organismo de cooperación internacional, compuesto por 37 estados. El informe PISA es un estudio llevado a cabo por la OCDE a nivel mundial que mide el rendimiento académico de los alumnos en matemáticas, ciencia y lectura, por sus siglas en inglés PISA significa Programme for International Student Assessment. 


\section{BIBLIOGRAFÍA}

Adick, C. (2018). Bereday and Hilker: origins of the 'four steps of comparison'model. Comparative Education, 54(1), 35-48. https://doi.org/10.1080/03050068.2017 .1396088

Balerdi, F. E., Larrañaga, J. G., Cartón, H. M., y Aranguren, E. A. (2018). Acogida del alumnado inmigrante recién llegado en la escuela inclusiva. El caso de Cataluña, País Vasco y Francia. RES: Revista de Educación Social, 27, 93-119.

Belvis, E., Moreno, M. V., y Ferrer, F. (2009). Los factores explicativos del éxito y fracaso académico en las universidades españolas, en los años del cambio hacia la convergencia europea. Revista española de educación comparada, 15, 61-92. http://revistas.uned.es/index.php/ REEC/article/view/7503

Bulman, G., y Fairlie, R. W. (2016). Technology and education: Computers, software, and the internet. In Handbook of the Economics of Education, (Vol. 5), 239280. Elsevier. https://doi.org/10.1016/ B978-0-444-63459-7.00005-1

Caamaño, D. P. (2009). Soporte tecnológico y gestión educativa de la inmigración. Teoría de la Educación. Educación y Cultura en la Sociedad de la Información. http://dx.doi.org/10.14201/eks.7521

Castañeda Quintero, L. J., y Adell, J. (2013). Entornos Personales de Aprendizaje: claves para el ecosistema educativo en red. Editorial Marfil. http://www.um.es/ ple/libro/

Caamaño, P. D. (2013). Familia, educación e inmigración. Un programa de intervención pedagógica. [Tesis doctoral]. Universidad de Santiago de Compostela.

Cebrián, A., Trillo, A., y González, A. (2019). PISA 2018. Programa para la Evaluación Internacional de los Estudiantes. Informe español. Ministerio de Educación.
Condeza, R., Bachmann, I., y Mújica, C. (2014). El consumo de noticias de los adolescentes chilenos: intereses, motivaciones y percepciones sobre la agenda informativa. Comunicar, 43(XXII), 55-64. http://dx.doi. org/10.3916/C43-2014-05

Coin, F. (2017). Does Enactive Teaching Improve Inclusion of Immigrant Pupils? A Research in Northern Italy's Schools. SAGE Open, 7(2). https://doi. org/10.1177/2158244017706403

Contucci, P., y Sandell, R. (2015). How integrated are immigrants? Demographic Research, 33, 1271-1280. https://doi. org/10.4054/DemRes.2015.33.46

Díaz-Vicario, A., Mercader, C., y Gairín, J. (2019). Uso problemático de las TIC en adolescentes. Revista Electrónica de Investigación Educativa, 21(e07), 1-11. https://doi.org/10.24320/redie.2019.21. e07.1882

Dryden-Peterson, S. (2016). Refugee education: The crossroads of globalization. Educational Researcher, 45(9), 473-482. https://doi. org/10.3102/0013189X16683398

Echazarra, A. (2018). How Has Internet Use Changed between 2012 and 2015? PISA in Focus No. 83. OECD Publishing. https:// doi.org/10.1787/22260919

Esteban, A. N. (2020). El derecho a la educación de los menores refugiados y solicitantes de asilo desde el enfoque basado en los derechos humanos: dificultades, finalidad e intervención educativa. Revista española de educación comparada, 35, 70-91. https://doi. org $/ 10.5944 /$ reec.35.2020.24342

Fuentes, M. D. C., y Gutiérrez, J. J. T. (2013). ¿Mejoran las TIC los resultados académicos de los estudiantes españoles? Extoikos, 9, 51-58.

Goicochea, M. A. D. (2008). El adolescente inmigrado latinoamericano en la escuela 
española. Las miradas docentes. Revista española de educación comparada, 14, 79-102.

González Merino, A. (2020). Panorama de la Educación 2019. Indicadores de la OCDE. Informe español. Versión preliminar. Ministerio de Educación.

Gurría, A. (2016). PISA 2015 resultados clave. OECD. https://www.oecd.org/ pisa/pisa-2015-results-in-focus-ESP.pdf

Huddleston, T., Niessen, J., y Tjaden, J. D. (2013). Using EU indicators of immigrant integration. Final Report for DirectorateGeneral for Home Affairs. Brussels: European Commission.

Hurwitz, L. B., y Schmitt, K. L. (2020). Can children benefit from early internet exposure? Short-and long-term links between internet use, digital skill, and academic performance. Computers $y$ Education,146, https://doi.org/10.1016/j. compedu.2019.103750

Irles, D. L., Perona, V. C., y Baños, Y. S. (2015). Relaciones entre hábitos de uso de videojuegos, control parental y rendimiento escolar. European journal of investigation in Health, Psychology and education, 3(3), 237-248. https://doi. org/10.30552/ejihpe.v3i3.58

Kogan, I. (2016). "Integration Policies and Immigrants' Labor Market Outcomes in Europe”. Sociological Science, 3, 335-358. https://doi.org/10.15195/v3.a16

Lieberman, D. A., y Gamst, G. (2015). Intercultural communication competence revisited: Linking the intercultural and multicultural fields. International Journal of Intercultural Relations, 48, 17-19. https://doi.org/10.1016/j. ijintrel.2015.03.007

Leicht, A., Heiss, J., y Byun, W. J. (2018). Issues and trends in education for sustainable development (Vol. 5). UNESCO Publishing. http://hdl.voced. edu.au/10707/451212

Manning, A., y Roy, S. (2010). Culture clash or culture club? National identity in
Britain. https://doi.org/10.1111/j.14680297.2009.02335.X

Marks, A. K., McKenna, J. L., y Coll, C. G. (2018). National immigration receiving contexts. European Psychologist, 17(2), 105-119. https://doi.org/10.1027/10169040/a000311

Martí Marco, M. R. (2019). Una introducción a los estudios de Educación Comparada en Alemania ("Vergleichende Erziehungswissenschaft”). https://doi. org/10.5944/reec.34.2019.24338

Martín-Gutiérrez, J., Mora, C. E., AñorbeDíaz, B., y González-Marrero, A. (2017). Virtual technologies trends in education. EURASIA Journal of Mathematics, Science and Technology Education, 13(2), 469-486. https://doi.org/10.12973/ eurasia.2017.00626a

Moledo, M. L., Rego, M. A. S., y Otero, A. G. (2012). Inmigración y educación. ¿Influye el nivel educativo de los padres en el rendimiento académico de los hijos? Teoría de la Educación. Revista Interuniversitaria, 24(2), 129-148. http://dx.doi.org/10.14201/10358

Mombelli, D. (2019). La metodología comparatista en los estudios literarios. https://doi.org/10.5944/ reec.34.2019.24379

OCDE (2016) OCDE Cooperación al Desarrollo Examen de Pares España $2016 \quad$ https://doi. org/10.1787/9789264260139-es

Rodicio-García, M. L., Ríos-de-Deus, M. P., Mosquera-González, M. J., y Abilleira, M. P. (2020). La brecha digital en estudiantes españoles ante la Crisis de la Covid-19. Revista Internacional de Educación para la Justicia Social, 9(3). https://doi. org/10.15366/riejs2020.9.3.006

Schachner, M. K. (2019). From equality and inclusion to cultural pluralismEvolution and effects of cultural diversity perspectives in schools. European Journal of Developmental Psychology, 
16(1), 1-17. https://doi.org/10.1080/1740 5629.2017.1326378

Secretaría General Técnica (2020). Datos y cifras Curso escolar 2019/2020 (2020). Subdirección General de Estadística y Estudios. Editorial Secretaría General Técnica. Centro de Publicaciones. Ministerio de Educación y Formación Profesional. https://sede.educacion. gob.es/publiventa/datos-y-cifrascurso-escolar-20192020/ensenanzaestadisticas/23109

Spörlein, C., y Schlueter, E. (2018). How education systems shape cross-national ethnic inequality in math competence scores: Moving beyond mean differences. PloS one, 13(3). https://doi.org/10.1371/ journal.pone.0193738

Terrén, E. (2007). Inmigración, diversidad cultural y globalización. Educación $e$ inmigración: nuevos retos para España en una perspectiva comparada. Centro de Investigaciones Sociológicas.
Torres Kompen, R., y Costa, C. (2013). Formación continua, aprendizaje a lo largo de la vida y PLEs. http://hdl. handle.net/10201/30412

Valero, D., y Viñas, T. P. (2019). Educación Inclusiva del Alumnado Inmigrante: Lecciones desde Estados Unidos, Canadá y Australia. Multidisciplinary Journal of Educational Research, 7(3), 316-338. $\quad$ https://doi.org/10.17583/ remie.2017.2946

Werbach, K., y Hunter, D. (2015). The gamification toolkit: dynamics, mechanics, and components for the win. Wharton School Press.

Zubíková, A. (2019, October). Integration of immigrants in the EU_15: success or failure? Proceedings of Economics and Finance Conferences (No. 10112410). International Institute of Social and Economic Sciences. https://doi. org/10.20472/EFC.2019.012.025

\section{PERFIL ACADÉMICO Y PROFESIONAL DE LA AUTORA}

Inés María González Vidal. Miembro del Grupo de Investigación en Tecnología Educativa (Tecnoeduc). Fue profesora de la Facultad de Contabilidad y Finanzas, Universidad de la Habana y profesora sustituta del Instituto de Computación, Universidad Federal de Alagoas. Actualmente vinculada al programa de doctorado Equidad e Innovación de la Facultad de Educación de la Universidad de Santiago de Compostela. Líneas investigación e-learning, hipermedia adaptativa, sistemas de información. https://orcid.org/0000-0003-0559-0321

E-mail: inesmaria.gonzalez@rai.usc.es.

Dirección: Programa de doctorado en Equidad e Innovación en Educación Universidad de Santiago de Compostela

Fecha de recepción del artículo: 29/07/2020

Fecha de aceptación del artículo: 25/08/2020

Fecha de aprobación para maquetación: 07/09/2020 